\title{
Enjoy your journey: the bergamot polyphenols from the tree to the cell metabolism
}

Salvatore Nesci ${ }^{1} \mathbb{D}$, Ernesto Palma², Vincenzo Mollace ${ }^{2^{*}}$, Giovanni Romeo ${ }^{3^{*}}$ and Francesca Oppedisano ${ }^{2}$

Keywords: Bergamot, Cell metabolism, Flavonoids, Antioxidant, Dyslipidemia, Metabolic diseases

The Mediterranean-style diet was hypothesized by Ancel Keys who observed in Southern Italy the highest concentration of centenarians in the world associated with a low incidence of coronary heart disease, and a lower incidence of cancer and adult-onset diabetes mellitus overall [1]. Since 2010, the Mediterranean diet (Md) has been labelled as an "intangible cultural heritage of humanity" with the acknowledged benefits on longevity and life health-related quality. The Md highlights that unsaturated fatty acids, vitamins, and polyphenols, associated with constant physical activity, are compounded with beneficial effects on human health [1].

Bergamot (Citrus bergamia Risso et Poiteau) is an endemic plant growing in the Southern Italian region of Calabria, containing different classes of glycosylated flavonoids (e.g., flavanones and flavones) a group of polyphenolic natural substances, that can exert beneficial effects to prevent disease [2]. The microclimate and soil of Jonic Calabria confer specific organoleptic characteristics to the bergamot juice. Determining bergamot DNA sequence $(\approx 300 \mathrm{Mbp})$ will have scientific importance for citrus taxonomy and phylogenetic tree reconstruction. Moreover, it will allow the development of a test for certification of bergamot fruit origin with obvious

\footnotetext{
*Correspondence: mollace@libero.it; egf.giovanni.romeo@gmail.com 2 Department of Health Sciences, Institute of Research for Food Safety \& Health (IRC-FSH), University "Magna Graecia" of Catanzaro, Catanzaro, Italy ${ }^{3}$ Medical Genetics Unit, Sant'Orsola-Malpighi University Hospital, Bologna, Italy

Full list of author information is available at the end of the article
}

consequences for the defence of the authentic fruit grown in Calabria.

The bergamot polyphenolic fraction (BPF), a particular product derived from bergamot juice, contains flavonoids, furocoumarin and other polyphenols. The main flavanone compounds are naringenin, hesperetin, eriodictyol glycoside, while the main flavones present are apigenin, luteolin, chrysoerol, diosmetin. Bergamot flavonoids can counteract dyslipidemia and hyperglycemia. Moreover, bergamot juice-derived food supplements, enriched with pectins and vitamin $\mathrm{C}$, can act on the adipose tissue feedback regulation and, according to the lipostat theory, significantly stimulate weight loss by increasing the levels of cardioprotective adiponectin, improve leptin and ghrelin levels, insulin sensitivity and reduce circulating insulin [3]. Since dyslipidemia is a well-established modifiable cardiovascular risk factor [4], the bergamot can have a homeostatic dose-dependent effect and potential synergistic action by statin administration on the human lipid profile. The main metabolism regulation effects are the total cholesterol decrease, triglycerides and low-density lipoprotein. Even if the bergamot supplementation molecular mechanism in dyslipidemia is not still understood, a possible explanation of cholesterol metabolism modulation is focused on its synthesis and faecal excretion. Therefore, the cellular targets are the 3-hydroxy-3-methyl-glutaryl-CoA (HMG$\mathrm{CoA}$ ) reductase, whose inhibition blocks the cholesterol endogenous synthesis, and the pancreatic cholesterolester-hydrolase and acyl-CoA cholesterol acyltransferase that are activated and, respectively, inhibited and fail original author(s) and the source, provide a link to the Creative Commons licence, and indicate if changes were made. The images or other third party material in this article are included in the article's Creative Commons licence, unless indicated otherwise in a credit line to the material. If material is not included in the article's Creative Commons licence and your intended use is not permitted by statutory regulation or exceeds the permitted use, you will need to obtain permission directly from the copyright holder. To view a copy of this licence, visit http://creativecommons.org/licenses/by/4.0/. The Creative Commons Public Domain Dedication waiver (http://creativeco mmons.org/publicdomain/zero/1.0/) applies to the data made available in this article, unless otherwise stated in a credit line to the data. 
esterification of endogenous cholesterol in human small intestinal mucosa by evading the cholesterol absorption [4].

In addition to the hypolipemic and hypoglycemic activities, the BPF guarantees antioxidant and anti-inflammatory effects [3]. Therefore, bergamot is effective in treating metabolic syndrome and has beneficial effects on the cardiovascular system. Consistently, an antioxidant response has been demonstrated both in vitro and in vivo. The effect leads to a reduction in cholesterol serum levels, glucose and triglycerides, a biological response accompanied by a reduction in systemic inflammation and, subsequently, an improvement in endothelial function. These effects were confirmed in patients treated with BPF, which showed a powerful effect in modulating the lipoproteins hepatic traffic and was able to counteract non-alcoholic liver disease (NAFLD). Specifically, brutieridin and melitidin have been shown to produce inhibition of HMG-CoA reductase. Recent data have also shown that BPF reduces cardiotoxicity induced by doxorubicin, counteracting the overproduction of reactive oxygen species (ROS), thus restoring protective autophagy and attenuating apoptosis and pathological myocardial remodelling [5]. Furthermore, mitochondrial dysfunction is the main event of ROS production in the cell, BPF promotes the correct coupling of the oxidative phosphorylation system and desensitizes the mitochondrial permeability transition pore formation, a phenomenon involved in triggering different forms of regulated cell death.

In addition, the BPF anti-inflammatory activity was tested in rats with cafeteria (CAF) diet-induced nonalcoholic steatohepatitis. The results obtained showed that BPF supplementation reduces the IL- 6 expression levels and increases the anti-inflammatory protein IL-10 expression with the reduction in liver inflammation. Rats fed with CAF and treated with BPF show a reduction in inflammatory foci. Furthermore, the antioxidant and anti-inflammatory effects of a phytocomplex, called Bergacyn, made of bergamot polyphenols and cynaropicrin isolated from artichoke (Cynara cardunculus), on patients with type 2 diabetes and NAFLD improved health condition, accordingly the phytocomplex action on oxidative stress and inflammation. Indeed, there was an increase in glutathione peroxidase and superoxide dismutase levels, whereas malondialdehyde and TNF- $\alpha$ levels were reduced by contributing to better NO-mediated reactive vasodilation [3].

Bergamot contains a plethora of molecules. Therefore, it will be interesting, in the nutraceuticals field, to be able to identify the molecules protagonists in the disease's prevention and the improvement of the cellular metabolism in pathological conditions. From a pharmacological point of view, it will be interesting to elucidate how the bergamot juice compounds act in a synergistic and/or additive way in carrying out their beneficial action on human health.

\section{Acknowledgements \\ Not applicable.}

\section{Authors' contributions}

SN and FO, conceptualization and writing —original draft; EP, validation and writing - review and editing; VM and GR, supervision and writing - review and editing. All authors read and approved the final manuscript.

Funding

Not applicable.

Availability of data and materials

Not applicable.

\section{Declarations}

Ethics approval and consent to participate

Not applicable.

Consent for publication

Not applicable.

Competing interests

The authors declare that they have no competing interests.

\section{Author details}

${ }^{1}$ Department of Veterinary Medical Sciences, University of Bologna, Ozzano Emilia, Italy. ${ }^{2}$ Department of Health Sciences, Institute of Research for Food Safety \& Health (IRC-FSH), University "Magna Graecia" of Catanzaro, Catanzaro, Italy. ${ }^{3}$ Medical Genetics Unit, Sant'Orsola-Malpighi University Hospital, Bologna, Italy.

Received: 5 October 2021 Accepted: 9 October 2021

Published online: 04 November 2021

References

1. Soldati L, Di Renzo L, Jirillo E, Ascierto PA, Marincola FM, De Lorenzo A. The influence of diet on anti-cancer immune responsiveness. J Transl Med. 2018;16:75.

2. Gardana C, Nalin F, Simonetti P. Evaluation of flavonoids and furanocoumarins from Citrus bergamia (bergamot) juice and identification of new compounds. Molecules. 2008;13:2220-8.

3. Carresi C, Gliozzi M, Musolino V, Scicchitano M, Scarano F, Bosco F, et al. The effect of natural antioxidants in the development of metabolic syndrome: focus on bergamot polyphenolic fraction. Nutrients. 2020;12:E1504.

4. Lamiquiz-Moneo I, Giné-González J, Alisente S, Bea AM, Pérez-Calahorra S, Marco-Benedí V, et al. Effect of bergamot on lipid profile in humans: a systematic review. Crit Rev Food Sci Nutr. 2020;60:3133-43.

5. Mollace V, Rosano GMC, Anker SD, Coats AJS, Seferovic P, Mollace R, et al. Pathophysiological basis for nutraceutical supplementation in heart failure: a comprehensive review. Nutrients. 2021;13:257.

\section{Publisher's Note}

Springer Nature remains neutral with regard to jurisdictional claims in published maps and institutional affiliations. 